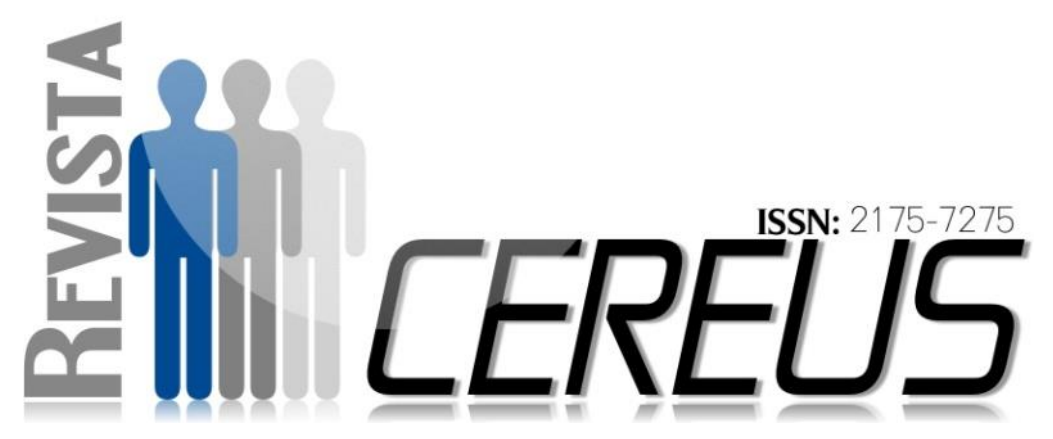

DOI:10.18605/2175-7275/cereus.v9n1p.118-131

\title{
ANÁLISE DA COMPLETUDE DOS DADOS SOBRE AS CAUSAS DE MORTALIDADE E MORBIDADE NO SISTEMA DE INFORMAÇÃO DE MORTALIDADE PARA O ESTADO DO TOCANTINS
}

RESUMO

\author{
SANTOS FILHO, Luciano Almeida dos ${ }^{1}$ \\ SILVA, Guilherme Morato ${ }^{2}$ \\ LO TURCO, Samanta Cunha ${ }^{2}$ \\ CARVALHO, Arthur Alves Borges de ${ }^{3}$
}

Introdução: O Serviço de Verificação de Óbitos (SVO) foi criado para elucidar causas de óbitos naturais mal definidas por meio de exames necroscópicos e registrar informações mais completas sobre as causas de morte no Sistema de Informação de Mortalidade (SIM). Objetivo: Analisar a completude dos dados sobre as causas de mortalidade e de morbidade no SIM do Tocantins. Método: Foram estudados 19780 registros de óbitos não fetais de residentes no Estado do Tocantins entre os anos de 2010, 2011 e 2012, obtidos por download do site do DATASUS do Ministério da Saúde.

\footnotetext{
1Discente de medicina da FAPAC/ITPAC em Porto Nacional, Tocantins. Monitor de Patologia Humana e membro integrante do Programa de Iniciação Científica. E-mail: luciano.filho10@hotmail.com ${ }^{2}$ Internos do $5^{\circ}$ ano de medicina da FAPAC/ITPAC em Porto Nacional, Tocantins.

${ }^{3}$ Médico Patologista. Mestre em Patologia Humana pela Faculdade de Medicina de Ribeirão Preto (USP). Docente da FAPAC/ITPAC em Porto Nacional, Tocantins, Brasil e da UNIRG em Gurupi, Tocantins, Brasil. Coordenador técnico do SVO, Palmas, Tocantins, Brasil.
} 
Resultados: A incompletude de dados registrados no SIM para o Estado do Tocantins foi superior a $10 \%$ e não houve diferença estatística significativa entre a completude de dados registrados pelo SVO e pelos demais atestantes. A análise da completude dos dados registrados no SIM, provenientes dos cinco atestantes permitiu observar que não houve diferença estatística na incompletude de dados entre os atestantes. Conclusão: a qualificação das informações sobre mortalidade, imprescindível à elaboração de políticas públicas sanitárias, persiste desafiadora no Tocantins. A criação de uma cultura da informação epidemiológica passa pela inserção curricular, educação e sensibilização médica continuada.

Palavras-chave: Estatísticas vitais. Causas de Morte. Serviços de Informação. Serviço de Verificação de Óbitos.

\section{ANALYSIS OF THE COMPLETENESS OF DATA ON MORTALITY CAUSES OF MORBIDITY AND MORTALITY IN INFORMATION SYSTEM FOR THE STATE OF TOCANTINS}

\section{ABSTRACT}

Introduction: Death Verification Service (SVO) was created to elucidate causes of natural deaths poorly defined by postmortem examinations and record more complete information on the causes of death in the Mortality Information System (SIM). Objective: To investigate the completeness of data on the causes of mortality and morbidity in Tocantins Mortality Information System, 2010-2012. Method: We studied 19780 no fetal death records of residents in the State of Tocantins between the years of 2010, 2011 and 2012, downloaded from the DATASUS of the Ministry of Health website. Results: The incompleteness of data recorded in the SIM for the State of Tocantins was higher than $10 \%$ and there 
was no statistically significant difference between the completeness of data recorded by the SVO and the other records. The analysis of the completeness of the data recorded in the SIM, from the five records showed that there was no statistical difference in the data incompleteness between the attesters. Conclusion: The qualification of information on mortality, essential for the elaboration of public health policies, remains challenging in Tocantins. The creation of a culture of epidemiological information involves the insertion of curriculum, education and continuous medical awareness.

Key Words: Vital statistics. Causes of Death. Information Services. Coroner's Service. 


\section{INTRODUÇÃO}

O sistema de informação de mortalidade (SIM) elabora estatísticas vitais para compilar uma série histórica de dados, estudarem as causas de mortes, comparam situações de saúde de diferentes áreas geográficas e promovem melhorias públicas sanitárias (SWAROOP apud LAURENTI; MELLO; GOTLIEB, 2006).

A coleta de dados para o SIM, realizada por municípios e estados da federação ocorre com o recolhimento da 1 a via da DO (Declaração de Óbitos) preenchida por médicos atestantes assistentes, substitutos, dos institutos médicos legais, dos serviços de verificação de óbitos (SVO) e outros. O preenchimento das DO constitui responsabilidade ética e jurídica do médico, como previsto nos artigos $82 \mathrm{e}$ 83 do Código de Ética Médica de 2009 (LUCENA, 2014).

Apesar de existirem poucos estudos sobre o impacto do SVO como fornecedor de dados ao SIM (NASCIMENTO et al, 2012), os SVO permitem a realização de diagnóstico isolado ou surtos de doenças emergentes, reemergentes e ainda agravos inusitados, bem como permite o aprimoramento da qualidade das informações sobre mortalidade $e$ morbidade (ELLINGER; BEZERRA, 2011).

Completude de dados representa o grau de preenchimento de variáveis ou campos analisados no SIM, mensurado pela presença de dados com distinção daquelas variáveis desprovidas de dados (FÉLIX et al, 2012). Os dados registrados no SIM são provenientes de informações médicas descritas nas DO.

A Organização Mundial de Saúde (OMS) relata que dados de mortalidade de muitos países permanecem não confiáveis para serem utilizados na elaboração de políticas públicas de saúde, persistindo urgente necessidade de melhoria nos sistemas de informação de mortalidade principalmente em países emergentes, a exemplo da América Latina (COLIN et al 2005; PAES, 2007).

A partir da análise de dados do SVO, pretende-se refletir e avaliar a expansão e a sustentabilidade de serviços médicos de interesse epidemiológico instituído pelo Ministério da Saúde. Nessa linha, o objetivo do estudo foi analisar a completude dos dados sobre as causas 
de mortalidade e de morbidade no SIM

\section{METODOLOGIA}

\section{Delineamento do estudo}

Estudo retrospectivo, transversal, quantitativo e qualitativo em série histórica de dados secundários em sistema de informação em saúde.

\section{Fonte de Dados e Variáveis do}

\section{Estudo}

O SIM consiste em software desenvolvido pelo Departamento de Informação de Saúde (DATASUS) com capacidade de consolidar, analisar e disponibilizar publicamente os dados sobre mortalidade em geral.

Foram selecionados 19.780 registros de óbitos de residentes do Tocantins no período de 2010 a 2012 SIM. Os quais estão disponíveis publicamente no site www2.datasus.gov.br (DATASUS, 2014) e foram coletados por meio de acesso virtual em agosto de 2014 .

\section{Para 0 estudo foram} selecionadas e analisadas as variáveis, linha $A$, linha $B$, linha $C$, linha $D$ e parte II. do Tocantins, 2010-2012.

A Linha A correspondente à variável sobre registros de causas de morte. As Linhas $B$ e Linha $C$ registros de causas intermediárias de óbitos. Linha $D$ registros de causas básicas de óbitos: A Parte II na DO apresenta duas linhas para descrição médica de causas de morbidades que contribuem para o óbito.

Os registros de óbitos fetais foram excluídos da análise, pois o SVO-TO não recebe óbitos fetais para exame necroscópicos em cumprimento da portaria 116 de fevereiro de 2009 (BRASIL, 2009a) e da resolução 1.779 do Conselho Federal de Medicina (CONSELHO FEDERAL DE MEDICINA, 2005). Foram excluídas as variáveis contendo dados subjetivos oriundos de informações descritivas da DO e que não apresentaram codificações no SIM.

\section{Análise da Completude de Dados Gerais}

A tabulação e análise descritiva dos dados foram realizadas no software Microsoft Excel 2013. Para verificar a 
existência da relação entre completude dos dados com o atestante, foi utilizado o teste de independência QuiQuadrado, considerando-se estatisticamente significativos aqueles com $P<0,05$. As análises de frequência relativa da média de variáveis com informações completas, dentro dos 19.780 registros e para os registros de cada um dos atestantes foram realizadas no software BioEstat 5.3.

\section{Análise da Completude de} Dados sobre Causas de Mortalidade e de Morbidade

Para analisar a completude das causas de mortalidade foram estudadas 4 variáveis no SIM: "Linha A, Linha B, Linha C, Linha D", que correspondem às informações contidas na DO sobre mortalidade, que foram transformadas em dados no SIM.

Os dados foram analisados quantitativamente (frequência absoluta e relativa) quanto à completude de variáveis com campos vazios ou não preenchidos para os registros de cada um dos cinco possíveis atestantes (assistentes, substitutos, IML, SVO e outros).

A Parte II é codificada em uma quantitativamente (frequência absoluta e relativa de campos vazios ou não preenchidos) para os registros de cada um dos cinco possíveis atestantes (assistentes, substitutos, IML, SVO e outros). O mesmo processo ocorreu para os casos nos quais os registros que não permitiram a identificação do atestante, pois a variável Atestante encontrava-se vazia ou não preenchida.

A avaliação qualitativa por capítulo da Classificação Internacional de Doenças (CID-10) das causas de mortalidade e de morbidade dentro dos registros do SIM foi prejudicada, direcionando o estudo para uma avaliação quantitativa sobre completude. Este redirecionamento ocorreu pela presença de completude, com um ou mais de um dado sobre mortalidade e morbidade nas variáveis A, B, C, D e Parte II.

\section{Aspectos Éticos}

Foram preservados os aspectos éticos da privacidade e da confidencialidade sem prejuízo a indivíduos ou às populações, de acordo com a Resolução CNS 466/12 do Conselho Nacional de Saúde que única variável, que foi analisada 
tratam de aspectos éticos em pesquisa (BRASIL,2012).

A pesquisa foi submetida ao Comitê de Ética e Pesquisa do Hospital das Clínicas da Faculdade de Medicina

\section{RESULTADOS}

Dentre os 19.780 registros analisados, foi possível discriminar os principais atestantes em 18.016, pois 1.764 exibiram a variável "atestante" vazia.

Dentre as linhas analisadas, a "Linha A" foi a que obteve menor quantidade de campos vazios (413 $2,1 \%$ ). A "Linha D" foi a variável com maior quantidade de campos vazios (15.225 76,9\%). As linhas B, C e Parte Il obtiveram campos vazios em 2.855 $(14,4 \%), \quad 7.733 \quad(39,1 \%)$ e 14.740 $(74,3 \%)$, respectivamente (Tabela 1 ).

Para o atestante "Assistente", que totalizou 6.942 registros, a linha $D$ foi a variável com maior número de campos vazios (5.266 - 75,9\%), de Ribeirão Preto que dispensou o estudo de avaliação ética por se tratar de pesquisa em dados secundários em software disponível e de livre acesso público via internet.

enquanto a linha A obteve menor número de campos vazios (164 - 2,3\%). Seguindo em ordem decrescente para as demais linhas: parte II com 4.996 (71,9\%) campos vazios, linha C com $2.752(39,6 \%)$ e linha B com 869 (12,5\%) (Tabela 2).

$\mathrm{O}$ atestante "Substituto" obteve 3.843 registros no total. Descrevendo os dados em ordem decrescente, a linha $D$ obteve o maior número de campos vazios, com 2.897 (75,3\%), seguido pela Parte II com 2.518 (65,5\%); 1.561 (40,6\%) para linha C; 491 (12,7\%) para linha B; e linha A com $125(3,2 \%)$, sendo a variável mais preenchida (Tabela 3 ).

Tabela 1 - Frequência absoluta e relativa de campos vazios no SIM, segundo causas de mortalidade e morbidade para o total de registros no SIM, Tocantins, Brasil, 2010 a 2012.

\begin{tabular}{lrr}
\multicolumn{1}{c}{ Variáveis } & № de campos vazios & Campos vazios (\%) \\
\hline Linha A & 413 & 2,1 \\
Linha B & 2.855 & 14,4 \\
Linha C & 7.733 & 39,1 \\
Linha D & 15.225 & 76,9 \\
Parte II & 14.740 & 74,3 \\
\hline
\end{tabular}

Rev. Cereus, v. 9, n. 1, p.118-131, jan-mai./2017, UnirG, Gurupi, TO, Brasil. 
Tabela 2 - Frequência absoluta e relativa de campos vazios no SIM, segundo causas de mortalidade e morbidade para o campo atestante Assistente, Tocantins, Brasil, 2010 a 2012.

\begin{tabular}{lrr}
\hline \multicolumn{1}{c}{ Variáveis } & № de Campos vazios & Campos vazios (\%) \\
\hline Linha A & 164 & 2,3 \\
Linha B & 869 & 12,5 \\
Linha C & 2.752 & 39,6 \\
Linha D & 5.266 & 75,9 \\
Parte II & 4.996 & 71,9 \\
\hline
\end{tabular}

Tabela 3 - Frequência absoluta e relativa de campos vazios no SIM, segundo causas de mortalidade e morbidade para o atestante Substituto, Tocantins, Brasil, 2010 a 2012.

\begin{tabular}{lrr}
\hline \multicolumn{1}{c}{ Variáveis } & No de Campos Vazios & Campos vazios (\%) \\
\hline Linha A & 125 & 3,2 \\
Linha B & 491 & 12,7 \\
Linha C & 1,561 & 40,6 \\
Linha D & 2.897 & 75,3 \\
Parte II & 2.518 & 65,5 \\
\hline
\end{tabular}

No total de 3.518 registros do atestante "IML", foi obtida como variável com maior número de campos vazios a Parte II (3.134 - 89,1\%), desviando do padrão apresentado pelos outros atestantes. Segue em ordem decrescente, as outras variáveis: linha D com 2.613 (74,3\%); $1.469(41,7 \%)$ para a linha C; linha B com $342(9,7 \%)$ e linha A como variável mais preenchida, com $26 \quad(0,7 \%)$ campos preenchidos (Tabela 4).

O atestante SVO com 818 registros apresentou a variável linha $D$ (662- 80,9\%) com menor número de campos preenchidos, seguido pela
Parte II com 564 (68,9\%); linha C com 495 (60,5\%); 271 (33,1\%) para linha B e a "linha A" foi a variável com menor número de campos vazios, com 14 $(1,7 \%)$ registros (Tabela 5$)$.

Dentre os registros do atestante "Outros" (2.895), a variável que se destacou por apresentar menor número de campos preenchidos foi a linha $D$ com $2.312(80,1 \%)$, dando sequência em ordem decrescente: Parte II (2.074 - 71,6\%); linha C com 1.456 (50,3\%); $545(18,8 \%)$ para a linha B e linha A seguindo o padrão de campo mais preenchidos, com 52 (1,7\%) (Tabela 6). 
Para os registros com o campo atestante vazio, sem discriminação de qual atestante registrou os dados, foram colhidos 1.764 registros. A linha D, seguindo, o padrão do total de atestantes, teve 1.466 campos vazios
$(83,1 \%)$, seguida pela Parte II com $1.454(82,4 \%)$. Seguem as demais variáveis: linha C com 900 (51\%); linha B com 337 (19,1\%) e linha A com 32 $(1,8 \%)$ (Tabela 7$)$.

Tabela 4 - Frequência absoluta e relativa de campos vazios no SIM, segundo causas de mortalidade e morbidade para o atestante IML, Tocantins, Brasil, 2010 a 2012.

\begin{tabular}{lrr}
\hline \multicolumn{1}{c}{ Variáveis } & № de Campos vazios & Campos vazios (\%) \\
\hline Linha A & 26 & 0,7 \\
Linha B & 342 & 9,7 \\
Linha C & 1.469 & 41,7 \\
Linha D & 2.613 & 74,3 \\
Parte II & 3.134 & 89,1 \\
\hline
\end{tabular}

Tabela 5 - Frequência absoluta e relativa de campos vazios no SIM, segundo causas de mortalidade e morbidade para o atestante SVO, Tocantins, Brasil, 2010 a 2012.

\begin{tabular}{lrr}
\hline \multicolumn{1}{c}{ Variáveis } & № de Campos vazios & Campos vazios (\%) \\
\hline Linha A & 14 & 1,7 \\
Linha B & 271 & 33,1 \\
Linha C & 495 & 60,5 \\
Linha D & 662 & 80,9 \\
Parte II & 564 & 68,9 \\
\hline
\end{tabular}

Tabela 6 - Frequência absoluta e relativa de campos vazios no SIM, segundo causas de mortalidade e morbidade para o atestante Outros, Tocantins, Brasil, 2010 a 2012.

\begin{tabular}{lrr}
\hline \multicolumn{1}{c}{ Variáveis } & № de Campos vazios & Campos vazios (\%) \\
\hline Linha A & 52 & 1,7 \\
Linha B & 545 & 18,8 \\
Linha C & 1.456 & 50,3 \\
Linha D & 2.312 & 80,1 \\
Parte II & 2.074 & 71,6 \\
\hline
\end{tabular}


Tabela 7 - Frequência absoluta e relativa de campos vazios no SIM, segundo causas de mortalidade e morbidade para o campo atestante Vazio, Tocantins, Brasil, 2010 a 2012.

\begin{tabular}{lrr}
\hline \multicolumn{1}{c}{ Variáveis } & № de Campos Vazios & Campos vazios (\%) \\
\hline Linha A & 32 & 1,8 \\
Linha B & 337 & 19,1 \\
Linha C & 900 & 51 \\
Linha D & 1.466 & 83,1 \\
Parte II & 1.454 & 82,4 \\
\hline
\end{tabular}

\section{DISCUSSÃO}

A incompletude de dados entre os atestantes não apresentou diferença estatística e as informações sobre a causa do óbito (linha A) demonstrou maior completude.

Para a OMS o controle de qualidade e a verificação do uso de informações em saúde, associados ao treinamento de recursos humanos são ações necessárias para o aprimoramento da informação (WHO, 2015). As incompetências e negligências de profissionais que lidam com óbitos parecem sustentar a problemática de completude, confiabilidade e credibilidade dos sistemas de informação em mortalidade nos países subdesenvolvidos (WHO, 2010).

A coleta dos dados sobre mortalidade para o SIM é permanente, com consolidação mensal por municípios, estados e consolidação anual em nível federal. A divulgação nacional dos dados é realizada anualmente, sob forma de anuários, boletins e CD-ROM (IBGE, 2016), entretanto durante a coleta de dados secundários para este estudo foi observada diferença quantitativa entre os registros de óbitos no SIM do Ministério da Saúde e os registros de óbitos no SIM para o Estado do Tocantins.

A diferença quantitativa de dados encontrada entre o SIM do Ministério da Saúde e o SIM para o Estado do Tocantins pode ser explicada pela persistência de captação de dados pelo sistema estadual, mesmo após a consolidação e o envio de dados ao sistema nacional. Ao pré-determinar este prazo de entrega de dados, o SIM do Ministério Rev. Cereus, v. 9, n. 1, p.118-131, jan-mai./2017, UnirG, Gurupi, TO, Brasil. 
da Saúde parece perder quantidade de registros que poderiam influenciar a quantidade e a qualidade da análise estatística nacional.

Ao avaliarmos a completude de dados registrados pelos cinco possíveis atestantes no SIM do Estado do Tocantins verificamos que a qualidade dos dados registrados no Sistema de Informações em Saúde do Ministério da Saúde pode corroborar com o relato da OMS quanto a baixa confiabilidade dos dados e estatísticas de mortalidade de países subdesenvolvidos

(RAMPATIGE, 2014).

A completude de dados dentro do SIM para o Estado do Tocantins registrado pelo SVO parece não diferir da completude de dados registrados pelos demais atestantes, pois 0 percentual da variável com maior completude e o percentual da variável com menor completude dentre os registros do SVO foram os mesmos percentuais das variáveis de maior e de menor completude dentre os registros dos demais atestantes.

De acordo com Mendonça, Drumond e Cardoso (2010), mesmo os médicos preocupando-se apenas com as informações sobre as causas de mortalidade e de morbidade permanece a dificuldade em sequenciar os eventos de modo cronológico desde a causa básica do óbito, passando pelas causas intermediárias e culminando na causa da morte.

Félix et al (2012) ao analisar a completude de dados do SIM no Espírito Santo sugeriu formação epidemiológica para profissionais de saúde em faculdades de medicina como medida para aprimorar a habilidades nos registros de dados em sistemas de saúde.

A habilidade no preenchimento da DO parece ser semelhante dentre os atestantes no Estado do Tocantins. A variável "Linha A" referente a causa do óbito apresentou maior completude e a variável "Linha D" referente à causa básica do óbito revelou menor completude, independente do tipo de atestante, em todos os registros analisados.

No estudo epidemiológico de 625 casos de suicídio em idosos, relatou que houvera redução da incompletude de dados quando a necropsia era utilizada para elucidação da causa básica (RIOS et al, 2013). Nos 19.780 registros analisados, a realização do exame necroscópico pareceu não aprimorar a completude de dados no SIM para IML e SVO, pois 
houve semelhança estatística quando comparados os dados sobre mortalidade e morbidade entre os atestantes "IML" e "SVO" com os dados de "substitutos", "assistentes" e "outros".

Esses resultados sugerem reflexões sobre a eficácia do SVO em

\section{CONCLUSÃO}

A habilidade e competência de registros de DO não diferem entre os atestantes no Tocantins. A baixa completude de dados no SIM pode comprometer a eficácia, a manutenção, e geram dúvida quanto à acreditação da atividade médica no SVO, e nas instituições de saúde onde trabalham médicos assistentes, substitutos e médicos legistas que representam os demais atestantes. melhorar as informações sobre mortalidade no Tocantins. Criado como instrumento de elucidação das causas mal definidas por meio de necropsias, esperava-se resultados mais positivos quanto completude de dados sobre mortalidade.
Educação médica continuada, fortalecimento do fluxo de informações entre instituições que lidam com óbitos podem fortalecer a elaboração e gestão de políticas públicas sanitárias. Essas ações visam sensibilizar o médico e devem ser implementadas com o objetivo de melhorar a qualidade do preenchimento da DO.

\section{REFERÊNCIAS}

AYRES, Manuel; AYRES Jr, Manuel; AYRES, Daniel Lima; SANTOS, Alex de Assis dos Santos. BioEstat: aplicações estatísticas nas áreas das ciências biológicas e médicas. Belém; Sociedade Civil Mamirauá: MCT-CNPq, 2007

AYRES, M. et al. BioEstat 5.3: Aplicações Estatísticas nas Áreas das Ciências Biológicas e Médicas. 5a ed. Belém-PA: Publicações Avulsas do Mamirauá, p. 361, 2011.

BRASIL. Ministério da Saúde. Portaria MS/GM o 116, de 11 de fevereiro de 2009. Regulamenta a coleta de dados, fluxo e periodicidade de envio das informações sobre óbitos e nascidos vivos para os Sistemas de Informações em Saúde sob gestão da 
Secretaria de Vigilância em Saúde. Diário Oficial da União da República Federativa do Brasil, Brasília, DF, 11 de fevereiro, 2009a.

BRASIL. Resolução CNS no 466, de 12 de dezembro de 2012. Aprova diretrizes e normas regulamentadoras de pesquisa envolvendo seres humanos. Diário Oficial da União. Brasília; 13 jun 2016. Seção 1, p. 59.

COLIN, M.; DORIS, M.F.; MIE, I.; CHALAPATI, R.; LOPES, D. Counting the dead and what they died from: an assessment of the global status of cause of death data. In: Bulletin of the World Health Organization; Past issues, v. 82, n. 3, 2005.

CONSELHO FEDERAL DE MEDICINA (Brasil). Resolução $\mathrm{n} \cong 1.179$, de 11 de novembro de 2005. Diário Oficial [da República Federativa do Brasil], Seção 1, Brasília - DF, p. 121, 5 dez. 2005.

DATASUS. Informações de Saúde. Disponível em: <http://www2.datasus.gov.br/DATASUS/index.php>. Acesso em: 27 ago. 2014.

ELLINGER, F.; BEZERRA, K.C.A. Manual de Procedimentos do Serviço de Verificação de Óbitos de Marília. Famema, p. 15, 2011.

FELIX, J. D. et al . Avaliação da completude das variáveis epidemiológicas do Sistema de Informação sobre Mortalidade em mulheres com óbitos por câncer de mama na Região Sudeste: Brasil (1998 a 2007). Ciênc. saúde coletiva, Rio de Janeiro , v. 17, n. 4, p. 945-953, Apr. 2012.

IBGE. Sistema de Informações de Mortalidade. 2016. Disponível em: $<$ http://ces.ibge.gov.br/base-de-dados/metadados/ministerio-da-saude/sistema-deinformacoes-de-mortalidade-sim.html>. Acesso em: 08 ago. 2016.

LAURENTI, R; MELLO, J.M.H.P.; GOTLIEB, S.L.D. O Sistema de Informações sobre Mortalidade: passado, presente e futuro. CBCD, São Paulo, p. 107, 2006.

LUCENA, L. et al. Declaração de Óbito: preenchimento pelo corpo clínico de um hospital universitário. Rev. bioét, v. 22, n. 2, p. 318-24, 2014.

MENDONÇA, F.M.; DRUMOND, E.; CARDOSO, A.M.P. Problemas no preenchimento da Declaração de Óbito: estudo exploratório. Revista Brasileira de Estudos de População, Rio de Janeiro, v. 27, n. 2, p. 285-295, jul./dez. 2010. 
NASCIMENTO, J.F.C.G.; GIL, J.L.A.; PASQUALUCCI, C.A; JACOB FILHO, W. Aspectos necrológicos do envelhecimento. Diagn. Tratamento, v. 17, n. 1, p. 58, 2012.

PAES, N. A. Qualidade das estatísticas de óbitos por causas desconhecidas dos Estados brasileiros. Rev. Saúde Pública, São Paulo, v. 41, n. 3, p. 436-445, jun. 2007.

RAMPATIGE, R.; MIKKELSEN, L.; HERNADEZ, I.R.; LOPEZ, A.D.; Systematic review of statistics on causes of deaths in hospitals: strengthening the evidence for policy-makers. Bull World Health Organ 2014;92:807-816 | doi: http://dx.doi.org/10.2471/BLT.14.137935.

RIOS, Marcela Andrade et al . Completude do sistema de informação sobre mortalidade por suicídio em idosos no estado da Bahia. J. bras. psiquiatr., Rio de Janeiro, v. 62, n. 2, p. 131-138, June 2013.

WHO, Health Information Systems, 2010. Disponível em: http://www.who.int/healthinfo/systems/WHO MBHSS 2010 section3 web.pdf

WHO Library Cataloguing-in-Publication Data Human resources for health information system: minimum data set for health workforce registry 2015. 1. Health Personnel. 2. Health Manpower. 3.Data Collection - standards. 4. Health Information Systems. 5.National Health Programs. I.World Health Organization. (c) World Health Organization 2015. ISBN 978 9241549226. 